\title{
Analysis of the FLC with Shrinking Span Membership Functions
}

\author{
Cheng-Liang Chen, Chung-Tyan Hsieh, Sheng-Nan Wang \\ Department of Chemical Engineering \\ National Taiwan University \\ Taipei 10617, TAIWAN ROC
}

\begin{abstract}
This article aims to investigate the input-output parametric relationship of a class of crisp-type fuzzy logic controllers (FLCS) using various t-norm-sum-gravity inference methods. Two most frequently used $t$-norms, namely the standard intersection and the algebraic product, are applied in calculating the matching level of each control rule, and the summation operator and the centerof-gravity method are applied to evaluate the final crisp output. The explicit mathematical forms of reasoning surfaces are addressed. Reasoning surfaces of these crisp-type FLCs are proved to be composed of a twodimensional multilevel relay, no matter which t-norm is used, and a local position-dependent nonlinear compensator with output pattern influenced by the t-norms selected.
\end{abstract}

\section{Introduction}

Until recently, investigations of theoretical aspects of fuzzy control systems are acknowledged a foundation of effective design of fuzzy logic controllers (FLCs). A good review of FLC design and a good collection of mathematical analyses for FLCs from a theoretic point of view are given in $[5,7,9]$. Some appealing FLC analyses are also reported in turn, such as $[11,12,2,8,6]$. These mathematical analyses provide clear description of input-output relations of the FLCs under some specific restrictions on membership functions, rule base, inference methods, etc. However, some impractical assumptions have been laid which have reduced the significance of these research works. For example, all the membership functions adopted in $[11,12,13,2]$ are equally-spaced triangles. It has been shown, however, that an FLC equipped with uniformly distributed membership functions is not effective for control $[6,2]$.

This article aims at generalizing the theoretical analyses given in the previous related researches $[11,12,2,8]$. Therein the crisp-type output membership functions and the triangular-shape input membership functions can be spaced nonuniformly, and two frequently used t-norms can be applied in evaluating the truth values of the control rules. The explicit input-output relations for the FLCs using these two t-norm-sum-gravity inference methods will be derived; the influence of applying various inference methods on output patterns will be studied and compared; It is expected that these analyses can lay rigorous foundations of effective FLC design and tuning.

\section{Topology of The Crisp-Type FLCs}

Suppose the input linguistic variables consist of error $(e)$ and change in error $(r)$ of the process output, and the output linguistic variable is the change in controller output $(u)$.

$$
\begin{aligned}
e_{m}(n T) & =\left.\{s p(t)-y(t)\}\right|_{t=n T} \\
r_{m}(n T) & =e_{m}(n T)-e_{m}(n T-T) \\
e^{*} & =G E e_{m}(n) \\
r^{*} & =G R r_{m}(n)
\end{aligned}
$$

The term sets, $T_{e}, T_{r}$ and $T_{u}$, are,

$$
\begin{aligned}
& T_{e}=\left\{E_{-m_{e}}, \cdots, E_{-1}, E_{0}, E_{1}, \cdots, E_{m_{e}}\right\} \\
& T_{r}=\left\{R_{-m_{r}}, \cdots, R_{-1}, R_{0}, R_{1}, \cdots, R_{m_{r}}\right\} \\
& T_{u}=\left\{U_{-m_{u}}, \cdots, U_{-1}, U_{0}, U_{1}, \cdots, U_{m_{u}}\right\}
\end{aligned}
$$

Where $I_{m_{*}}=\left\{-m_{*}, \cdots,-1,0,1, \cdots, m_{*}\right\}$ is the index set. Let $E_{i}^{*}$ 's, $R_{j}^{*}$ 's, and $U_{k}^{*}$ 's, be three series of principal values (cores) distributed on $U_{e}, U_{r}$ and $U_{u}$ 
with unequal spacings,

$$
\begin{aligned}
& E_{-m_{e}}^{*}<\ldots<E_{-1}^{*}<E_{0}^{*}=0<E_{1}^{*}<\ldots<E_{m_{e}}^{*} \\
& R_{-m_{r}}^{*}<\ldots<R_{-1}^{*}<R_{0}^{*}=0<R_{1}^{*}<\ldots<R_{m_{r}}^{*} \\
& U_{-m_{u}}^{*}<\ldots<U_{-1}^{*}<U_{0}^{*}=0<U_{1}^{*}<\ldots<U_{m_{u}}^{*}
\end{aligned}
$$

The output membership functions, $U_{k}(u)$ 's, are all in singleton forms (i.e., crisp-type),

$$
U_{k}(u)= \begin{cases}1 & \text { at } u=U_{k}^{*} \\ 0 & \text { otherwise }\end{cases}
$$

The input membership functions, $E_{i}(e)$ 's $\left(R_{j}(r)\right.$ 's) are composed of series of unequally-spaced triangles,

$E_{i}(e)=\left\{\begin{array}{cl}\frac{e-E_{i-1}^{*}}{E_{i}^{*}-E_{i-1}^{*}} & \text { for } E_{i-1}^{*} \leq e \leq E_{i}^{*} \\ \frac{E_{i+1}^{*}-e}{E_{i+1}^{*}-E_{i}^{*}} & \text { for } E_{i}^{*} \leq e \leq E_{i+1}^{*} \\ 0 & \text { others }\end{array} \quad\right.$ for $i \in I_{m_{e}}$

Note that when these $E_{i}^{*}$ 's, $R_{j}^{* \prime}$ 's and $U_{k}^{* \prime}$ s are ordered in a more specific way such that $E_{i}^{*}=$ $\frac{i}{m_{e}} s_{e}^{m_{e}-|i|}, R_{j}^{*}=\frac{j}{m_{r}} s_{r}^{m_{r}-|j|}$ and $U_{k}^{*}=\frac{k}{m_{u}} s_{u}^{m_{u}-|k|}$, where $s_{e}, s_{r}, s_{u} \in(0,1]$ are the shrinking factors for $e, r$ and $u$, these USMFs would be reduced into series of shrinking-span membership functions (SSMFs for short) [1]. The SSMFs could be further reduced into equally-spaced membership functions (ESMFs for short) if they have unity shrinking factors $\left(s_{e}=s_{r}=s_{u}=1\right)$, and in such a case the spacings would become $\frac{1}{m_{e}}, \frac{1}{m_{r}}$, and $\frac{1}{m_{u}}$, respectively. The appearance of typical USMFs, SSMFs, and ESMFs can be found elsewhere [4]. An FLC equipped with singleton output MFs and USMFs (SSMFs, ESMFs) for inputs is named the crisp-type USMFs-FLC (SSMFs-FLC, ESMFs-FLC).

In this study, the simple control rule mapping [10] is used in the rule base to simplify the analysis.

$$
R_{i, j}: \text { IF } e \text { is } E_{i} \text { AND } r \text { is } R_{j} \text { THEN } u \text { is } U_{k=i+j}
$$

We assume $m_{e}=m_{r}=m$ and therefore $m_{u}=m_{e}+$ $m_{r}=2 m$ to simply the analysis furthermore. A series of fuzzy operators, $\mathbb{F}, \mathbb{I}, \mathbb{A}$, and $\mathbb{D}$, are used to perform the decision making in the FLC: $\mathbb{F}$ calculates the firing level in each of the rules in the rule base; II and $\mathbb{A}$ perform the implication and aggregation of the FLC, respectively; and $\mathbb{D}$ converts the aggregated output fuzzy set $U(u)$ into a single crisp output value $u^{*}$.

$$
\begin{aligned}
\phi_{i, j} & =\mathbb{F}\left(E_{i}\left(e^{*}\right), R_{j}\left(r^{*}\right)\right) \\
U(u) & =\mathbb{A}\left(\mathbb{I}\left(\phi_{i, j}, U_{k=i+j}(u)\right)\right) \\
u^{*} & =\mathbb{D}(U(u)) \\
u_{m}(n T) & =\operatorname{GU} u^{*} \\
\operatorname{CO}(n T) & =\operatorname{CO}(n T-T)+u_{m}(n T) \\
\operatorname{CO}(t) & =\operatorname{CO}(n T) \text { for } t \in[n T, n T+T]
\end{aligned}
$$

In this article, the summation is selected as $\mathbb{A}$, the center of area (COA) method is used as $\mathbb{D}$. As for the $\mathbb{F}$ operator, some frequently used $t$-norms are applied and compared. Such a class of fuzzy controllers can be named as the crisp-type FLCs using various t-norm-sum-gravity inference methods. The final crisp output $u_{m}$ for such crisp-type FLCs is $[11,8]$ :

$$
u_{m}=\mathrm{GU} \times u^{*}=\mathrm{GU} \times \frac{\sum_{\phi_{i, j} \neq 0} \phi_{i, j} U_{i+j}^{*}}{\sum_{\phi_{i, j} \neq 0} \phi_{i, j}}
$$

\section{Analysis of the Crisp-Type FLCs}

\subsection{Input-output relation of the crisp-type FLCs}

For the crisp-type USMFs-FLCs depicted in section 2 , it is obvious that only four control rules are significant, i.e., having non-zero firing levels, for input values $e^{*} \in\left[E_{i}^{*}, E_{i+1}^{*}\right]$ and $r^{*} \in\left[R_{j}^{*}, R_{j+1}^{*}\right]$. They are [11],

(r1) if $e$ is $E_{i+1}$ and $r$ is $R_{j+1}$ then $u$ is $U_{k+2}$;

(r2) if $e$ is $E_{i+1}$ and $r$ is $R_{j}$ then $u$ is $U_{k+1}$;

(r3) if $e$ is $E_{i}$ and $r$ is $R_{j+1}$ then $u$ is $U_{k+1}$;

(r4) if $e$ is $E_{i}$ and $r$ is $R_{j}$ then $u$ is $U_{k}$.

For various combinations of crisp input values $e^{*}$ and $r^{*}$ of the USMFs-FLC, the ijth-block is used to refer the rectangular region in which $e^{*} \in\left[E_{i}^{*}, E_{i+1}^{*}\right] \subset$ $U_{e}$ and $r^{*} \in\left[R_{j}^{*}, R_{j+1}^{*}\right] \subset U_{r}$ such as shown in figure 1. The $i j$ th-block, where $i, j \in I_{m}-\{m\}$, can be normalized furthermore into a unit square by defining the normalized input values $\mathcal{E}=\frac{e^{*}-E_{i}^{*}}{E_{i+1}^{*}-E_{i}^{*}}$ and $\mathcal{R}=\frac{r^{*}-R_{j}^{*}}{R_{j+1}^{*}-R_{j}^{*}}$. Then the nonzero membership values are $E_{i+1}\left(e^{*}\right)=\mathcal{E}, E_{i}\left(e^{*}\right)=1-\mathcal{E}, R_{j+1}\left(r^{*}\right)=\mathcal{R}$, and $R_{j}\left(r^{*}\right)=1-\mathcal{R}$. According to the relative magnitude of the membership values, four regions are 
Figure 1 The ijth-block for $e$ and $r$ on the domain $\underline{U_{e} \times U_{r}}$

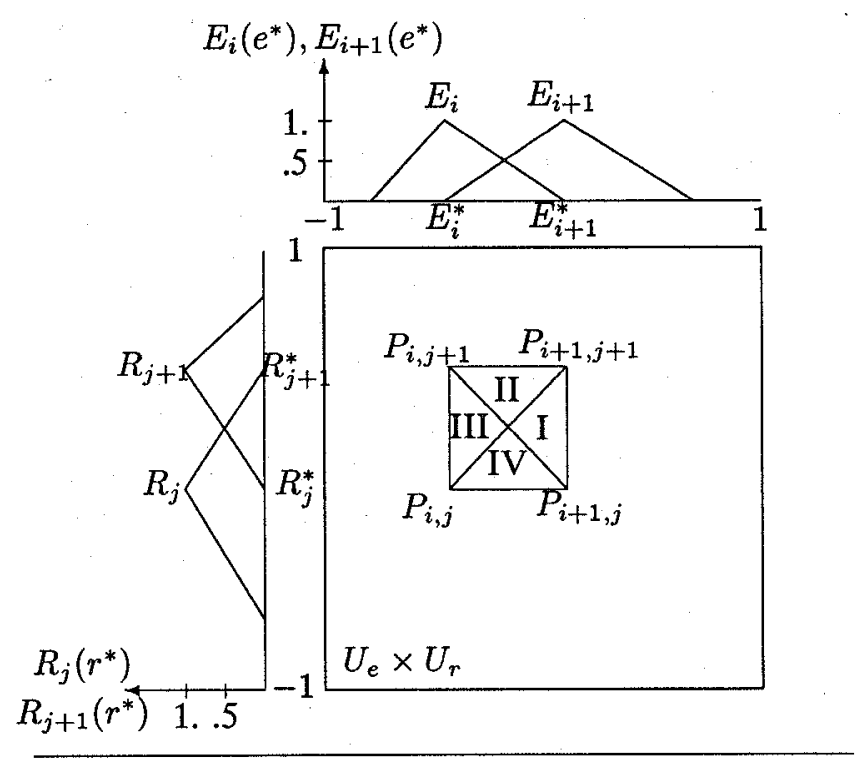

Figure 2 The normalized $i j$ th-block for all $i, j \in I_{m}-$ $\{m\}$ and the relative magnitudes of normalized inputs in the four regions

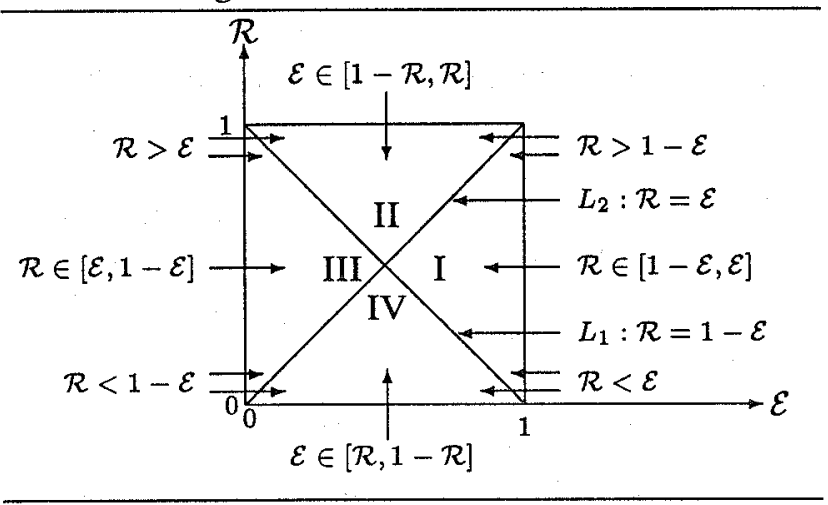

partitioned by lines $L_{1}: \mathcal{E}+\mathcal{R}=1$ and $L_{2}: \mathcal{E}=\mathcal{R}$ to represent the different combinations of the membership values such as depicted in figure 2 .

The relative magnitude of concerned membership values are used to calculate the firing levels of the control rules such as listed in Table 1. Here two $\mathrm{t}$-norms frequently used as fuzzy intersections are applied, namely, the standard intersection (the maximum $t$-norm, denoted as $\underset{\max }{\mathbb{T}}$ ) and the algebraic product (denoted as $\underset{a p}{\mathbb{T}}$ ). Applying the firing levels in different situations listed in table 1 , the explicit mathematical form of final output $\mathrm{GU} \times u^{*}$ of the crisp-type USMFs-FLC when applying vari-
Table 1 The firing levels for rules $1 \sim 4$ in the $i j t h$ block of the USMFs-FLC

\begin{tabular}{|c|c|c|c|c|c|}
\hline & & $\mathrm{r1}$ & $\mathrm{r} 2$ & $\mathrm{r} 3$ & $\mathrm{r4}$ \\
\hline \multirow{4}{*}{$\underset{\max }{\mathbb{T}}$} & I & $\mathcal{R}$ & $1-\mathcal{R}$ & $1-\mathcal{E}$ & $1-\mathcal{E}$ \\
\hline & II & $\mathcal{E}$ & $1-\mathcal{R}$ & $1-\mathcal{E}$ & $1-\mathcal{R}$ \\
\hline & III & $\mathcal{E}$ & $\mathcal{E}$ & $\mathcal{R}$ & $1-\mathcal{R}$ \\
\hline & IV & $\overline{\mathcal{R}}$ & $\mathcal{E}$ & $\overline{\mathcal{R}}$ & $1-\mathcal{E}$ \\
\hline$\underset{a p}{\mathbb{T}}$ & IV & $\mathcal{E R}$ & $\mathcal{E}(1-\mathcal{R})$ & $(1-\mathcal{E}) \mathcal{R}$ & $(1-\mathcal{E})(1-\mathcal{R})$ \\
\hline
\end{tabular}

ous t-norm-sum-gravity inference methods can be addressed.

(Theorem 1) The final output value of the crisp-type USMFs-FLCs in the ijth-block, no matter which $t$ norm is applied, is bounded by the minimum and the maximum output principal values of the ijth-block.

$$
U_{i+j}^{*} \leq u^{*} \leq U_{i+j++2}^{*}
$$

(Theorem 2〉 For any given input pattern in the ijthblock $e^{*} \in\left[E_{i}^{*}, E_{i+1}^{*}\right]$ and $r^{*} \in\left[E_{j}^{*}, R_{j+1}^{*}\right]$, let $\mathcal{E}=\frac{e^{*}-E_{i}^{*}}{E_{i+1}^{*}-E_{i}^{*}}$ and $\mathcal{R}=\frac{r^{*}-R_{j}^{*}}{R_{j+1}^{*}-R_{j}^{*}}$ denote the normalized input values in the normalized ijth-block. Then the crisp output of the crisp-type USMFs-FLC in the ijthblock using any t-norm-sum-gravity inference method, $u_{m}=G U \times u^{*}$, is the sum of a global two-dimensional multilevel relay, $G U \times u_{G}^{*}$, and a t-norm dependent local nonlinear compensator, $G U \times u_{L}^{*}:(k=i+j)$

$$
\begin{aligned}
& u_{m}=G U \times u^{*}=G U \times\left(u_{G}^{*}+u_{L}^{*}\right)=u_{G}+u_{L} \\
& u_{G}^{*}=U_{k+1}^{*} \\
& u_{L}^{*}=\left(u_{L}^{*}\right)_{\dagger} \quad \dagger \in\{\underset{\text { min } a p}{\mathbb{T}}, \underset{T}{\mathbb{T}}, P L\}
\end{aligned}
$$$$
\left(u_{L}^{*}\right)_{m a x}^{\mathbf{T}}= \begin{cases}\frac{\mathcal{R}\left(U_{k+2}^{*}-U_{k+1}^{*}\right)-(1-\mathcal{E})\left(U_{k+1}^{*}-U_{k}^{*}\right)}{1+2(1-\mathcal{E})} & (I) \\ \frac{\mathcal{\varepsilon}\left(U_{k+2}^{*}-U_{k+1}^{*}\right)-(1-\mathcal{R})\left(U_{k+1}^{*}-U_{k}^{*}\right)}{1+2(1-\mathcal{R})} & (I I) \\ \frac{\mathcal{E}\left(U_{k+2}^{*}-U_{k+1}^{*}\right)-(1-\mathcal{R})\left(U_{k+1}^{*}-U_{k}^{*}\right)}{\mathcal{R}\left(U_{k+2}^{*}-U_{k+1}^{*}\right)-(1-\mathcal{E})\left(U_{k+1}^{*}-U_{k}^{*}\right)} & (I I I) \\ \frac{1+2 \mathcal{R}}{1+2} & (I V)\end{cases}
$$$$
\begin{aligned}
\left(u_{L}^{*}\right)_{\mathfrak{a} p}= & (\mathcal{E})(\mathcal{R})\left(U_{k+2}^{*}-U_{k+1}^{*}\right) \\
& -(1-\mathcal{E})(1-\mathcal{R})\left(U_{k+1}^{*}-U_{k}^{*}\right)
\end{aligned}
$$$$
\left(u_{L}^{*}\right)_{P L}= \begin{cases}(\mathcal{E}+\mathcal{R}-1)\left(U_{k+2}^{*}-U_{k+1}^{*}\right) & (I, I I) \\ (\mathcal{E}+\mathcal{R}-1)\left(U_{k+1}^{*}-U_{k}^{*}\right) & (I I I, I V)\end{cases}
$$

where the piecewise linear output, $\left(u_{L}^{*}\right)_{\mathrm{PL}}$, is artificially synthetized by linear interpolation from outputs on the four corners of the ijth-block. 


\section{Remarks:}

1. The role of the local output is to compensate the discrete-type global output so as to lead to a continuous final reasoning surface. Different $t$ norms selected as the firing operator would give various compensation behaviors.

2. The Lipschitz condition of the reasoning surface guarantees its uniform continuity, and this is a basic requirement for a potentially effective controller [6]. The standard intersectionsum-gravity method and the algebraic productsum-gravity method are admissible since they can manufacture Lipschitz functions at least. Though the simple control rules have been assumed in this article to simplify the analysis, both inference methods have the potential to provide the required nonlinear reasoning surface and the better control performance over the linear controllers if suitable tuning parameters and control rules are adopted.

3. These explicit mathematical forms of reasoning surfaces reveal that the nonlinearity of the FLCs using the standard intersection comes from the denominators, whereas the cross product term of $\mathcal{E}$ and $\mathcal{R}$ leads to the nonlinear nature when algebraic product is used. The local output of the FLC in the $i j$ th-block using $\underset{a p}{\mathbb{T}}$ can be rearranged into combinations of the nonlinear and the linear parts,

$$
\begin{aligned}
\left(u_{L}^{*}\right)_{\mathbb{a p}}= & \mathcal{E R}\left[\left(U_{k+2}^{*}-U_{k+1}^{*}\right)-\left(U_{k+1}^{*}-U_{k}^{*}\right)\right] \\
& +(\mathcal{E}+\mathcal{R}-1)\left(U_{k+1}^{*}-U_{k}^{*}\right)
\end{aligned}
$$

This output equation becomes a linear one in the $i j$ th-block if the singleton output membership functions are equally-spaced, i.e., $U_{k+2}^{*}-U_{k+1}^{*}=$ $U_{k+1}^{*}-U_{k}^{*}=\frac{1}{2 m} \forall k \in I_{2 m}-\{2 m-1,2 m\}$. The overall reasoning surface would be made up of a set of piecewise linear rectangles. Furthermore, the FLC using algebraic product would be equivalent to a linear PI controller if the singleton output MFs and the triangular input MFs are all equally-spaced.

4. The effects of shrinking factors on the reasoning surfaces can be found elsewhere [3].

\subsection{The relative degree-of-nonlinearity}

Theorem 2 implies that the crisp-type USMFsFLC is nonlinear in nature. We have synthetized the piecewise linear output surface over each block in the input domain. A relative degree-of-nonlinearity can be defined as the discrepancy between outputs using different $t$-norms and the piecewise linear one.

$$
\begin{aligned}
\varrho_{\dagger} & \equiv \max _{\forall i, j \in I_{m}-\{m\}} \frac{\iint_{i j \text { th-block }}\left|u_{\dagger}^{*}-u_{\mathrm{PL}}^{*}\right| d r d e}{\left(E_{i+1}^{*}-E_{i}^{*}\right)\left(R_{j+1}^{*}-R_{j}^{*}\right)} \\
& =\max _{\forall i, j \in I_{m}-\{m\}} \iint_{i j \text { nomulized }}\left|u_{\dagger}^{*}-u_{\mathrm{PL}}^{*}\right| d \mathcal{R} d \mathcal{E} \\
\dagger & \in\{\underset{\text { max }}{\mathbb{T}}, \underset{a p}{\mathbb{T}}\}
\end{aligned}
$$

From the definition of $\varrho_{+}$, the higher the value of $\varrho_{\dagger}$ the higher the degree of nonlinearity, and $\varrho_{\dagger}=0$ indicates that the FLC a piecewise linear controller. For the SSMFs-FLC, the $\varrho_{\dagger}$ 's should be evaluated for $i=j=m-1$. The effects of the input shrinking factors and the number of linguistic terms on the relative degree-of-nonlinearity defined above can be summarized in the following theorem.

$\langle$ Theorem 3) Let

$$
\begin{aligned}
& \alpha \equiv U_{2 m}^{*}-U_{2 m-1}^{*}=1-\left(1-\frac{1}{2 m}\right) s_{u} \\
& \beta \equiv U_{2 m-1}^{*}-U_{2 m-2}^{*}=\left(1-\frac{1}{2 m}\right) s_{u}-\left(1-\frac{1}{m}\right) s_{u}^{2}
\end{aligned}
$$

Then the relative degree-of-nonlinearity for crisp-type SSMFs-FLCs using various t-norm-sum-gravity inference methods defined above are

$$
\begin{aligned}
\varrho_{\mathrm{T}}= & \frac{\alpha-\beta}{12} \\
\varrho_{\max }^{\mathrm{T}}= & \frac{24 \alpha^{3}+31 \alpha^{2} \beta+12 \alpha \beta^{2}+\beta^{3}}{24 \alpha^{2}} \\
& -\alpha\left(\frac{3 \alpha+\beta}{2 \alpha}\right)^{2} \ln \left(\frac{3 \alpha+\beta}{2 \alpha}\right)
\end{aligned}
$$

\subsection{The ultimate behavior with infinite linguistic terms}

The following theorems further point out the ultimate behavior of the crisp-type SSMFs-FLC and ESMFs-FLC, respectively, as the number of linguistic terms approaches infinity, $m \rightarrow \infty$. 
(Theorem 4) For a crisp-type SSMFs-FLC using any t-norm-sum-gravity inference method, the crisp output on the ijth-block is bounded by two linear PI controllers as the number of linguistic terms approaches infinity.

$$
K_{p}^{\ell}\left(r_{m}+\frac{T}{\tau_{I}^{\ell}} e_{m}\right) \leq u_{m} \leq K_{p}^{u}\left(r_{m}+\frac{T}{\tau_{I}^{u}} e_{m}\right)
$$

where

$$
\begin{aligned}
K_{p}^{\ell} & =\frac{G U \times G R}{2} \frac{s_{u}^{2 m-|i+j|}}{s_{r}^{m-|j+1|}} \\
K_{p}^{u} & =\frac{G U \times G R}{2} \frac{s_{u}^{2 m-|i+j+2|}}{s_{r}^{m-|j|}} \\
\tau_{I}^{\ell} & =\frac{G R}{G E} \frac{s_{e}^{m-|i+1|}}{s_{r}^{m-|j+1|}} T \\
\tau_{I}^{u} & =\frac{G R}{G E} \frac{s_{e}^{m-|i|}}{s_{r}^{m-|j|}} T
\end{aligned}
$$

(Theorem 5) The crisp-type ESMFs-FLC using any tnorm-sum-gravity inference method is equivalent to a linear $P I$ controller with $K_{p}=\frac{\mathrm{G} U \times G R}{2}$ and $\tau_{I}=\frac{G R}{G E} T$ as the number of linguistic terms approaches infinity.

\section{Conclusion}

This article studied the input-output parametric relationship of a class of crisp-type fuzzy logic controllers using various t-norm-sum-gravity inference methods. The explicit mathematical expressions of reasoning surfaces for the crisp-type FLCs using different t-norms as the firing operator were addressed. The reasoning outputs of these crisp-type FLCs were decomposed into two parts: a two-dimensional multilevel relay which is independent of the $t$-norms used, and a local nonlinear compensator with different output patterns according to the t-norm selected. A measure of relative degree-of-nonlinearity was defined and used to examine the output figures of these crisp-type FLCs using various t-norms.

\section{References}

[1] C. L. Chen and C. T. Hsieh. User-friendly design method for fuzzy logic controller. IEE Proc. Control Theory And Applications, in press, 1996.

[2] C. L. Chen and F. C. Kuo. design and analysis of a fuzzy logic controller. Int. J. Systems Sci, 26(5):1223$1248,1995$.
[3] C. L. Chen, S. N. Wang, C. T. Hsieh, and F. Y. Chang. Theoretical analysis of the crisp-type fuzzy logic controllers using various $t$-norm-sum-gravity inference methods. submitted to IEEE Trans. on Fuzzy Systems, 1996.

[4] C. L. Chen, S. N. Wang, C. T. Hsieh, and F. Y. Chang. Theoretical analysis of the FLC with unequally-spaced triangular membership functions. submitted to Fuzzy Sets and Systems, 1996.

[5] C. C. Lee. Fuzzy logic in control systems: fuzzy logic controller, part I, II. IEEE Transactions on Systems, Man, and Cybernetics, 20(2):404-435, March/April 1990.

[6] F. L. Lewis and K. Liu. Towards a paradigm for fuzzy logic control. Automatica, 32(2):167-181, 1996.

[7] H. T. Nguyen, M. Sugeno, R. Tong, and R. R. Yager. Theoretical aspects of fuzzy control $(E d$.$) . John Wiley$ \& Sons, Inc., New York, 1995.

[8] W. Qiao and M. Mizumoto. PID type fuzzy controller and parameters adaptive method. Fuzzy Sets and Systems, 78:23-35, 1996.

[9] L.-X. Wang. Adaptive fuzzy systems and control: design and stability analysis. Prentice-Hall International, Inc., New Jersey, 1994.

[10] C. Wong, C. Chou, and D. Mon. Studies on the output of fuzzy controller with multiple inputs. Fuzzy Sets and Systems, 57:149-158, 1993.

[11] H. Ying. A nonlinear fuzzy controller with linear control rules is the sum of a global two-dimensional multilevel relay and a local nonlinear proportionalintegral controller. Automatica, 29:499-505, 1993.

[12] H. Ying. General analytical structure of typical fuzzy controller and their limiting structure theorems. Automatica, 29:1139-1143, 1993.

[13] H. Ying. The simplest fuzzy controllers using different inference methods are different nonlinear proportional-integral controllers with variable gains. Automatica, 29:1579-1589, 1993. 